\title{
Changes in pancreatic cancer mortality, period patterns, and birth cohort patterns in Japan: analysis of mortality data in the period 1968-2002
}

\author{
Takayuki Seino $\cdot$ Hiroto Nakadaira $\cdot$ \\ Kazuo Endoh · Masaharu Yamamoto
}

Received: 19 December 2007/Accepted: 3 April 2008/Published online: 24 May 2008

(C) The Japanese Society for Hygiene 2008

\begin{abstract}
Objectives The 5-year survival rate for pancreatic cancer is known to be lower than that for cancer at any other site in the body, and the proportion of pancreatic cancer deaths among all cancer deaths has been increasing in Japan. The aim of this study was to investigate pancreatic cancer mortality in the light of temporal and geographical trends in the 47 prefectures of the country between 1968 and 2002.

Methods To survey the geographical aspects of pancreatic cancer mortality, we mapped the direct age-adjusted mortality rates of persons aged 40 years and older by sex in seven 5-year periods (1968-1972 to 1998-2002). We also evaluated the changes in period and birth cohort trends using estimable functions based on the age-period-cohort models in each prefecture.

Results During the observation period the Hokkaido and Tohoku regions had high mortality rates for both sexes. No significant increase in period trends was observed from 1973 to 2002, but significant increases in cohort trends
\end{abstract}

T. Seino $(\bowtie) \cdot$ M. Yamamoto

Department of Community Preventive Medicine,

Niigata University Graduate School of Medical

and Dental Sciences, 757 Asahimachi-dori,

Ichi-bancho, Niigata 951-8510, Japan

e-mail: setaka@msj.biglobe.ne.jp

\section{H. Nakadaira}

Faculty of Nursing, Social Welfare, and Psychology Department of Nursing,

Niigata Seiryo University, Niigata, Japan

\section{K. Endoh}

Department of Health and Nutrition,

School of Medical Technology,

Niigata University of Health and Welfare,

Niigata, Japan were observed from 1913 to 1962 - in two prefectures, for males, and in four prefectures, for females.

Conclusions The results of this study reveal a combination of time trends in pancreatic cancer mortality and changes in period or birth cohort trends. The changes in cohort trends in each prefecture were more variable than the period trends. This finding probably indicates the need for further investigation of the cohort-related factors involved in the prevalence of pancreatic cancer. Further research on mortality in the 47 prefectures needs to be conducted while taking the two time effects into account.

Keywords Age-period-cohort model $\cdot$ Cohort effect . Estimable functions - Pancreatic cancer - Period effect

\section{Introduction}

Pancreatic cancer is generally characterized as being difficult to diagnose and having a poor prognosis. The 5-year survival rate for pancreatic cancer is known to be the lowest of all cancers [1], and in recent years the proportion of deaths from pancreatic cancer has been increasing. In 1993, 8139 male patients died from pancreatic cancer, accounting for $5.7 \%$ of all cancer deaths among males; among females with pancreatic cancer, 6574 patients died, accounting for $7.0 \%$ of all cancer deaths among females [2]. In 2003, however, the corresponding figures were $11,280(6.0 \%)$ for males and $9868(8.0 \%)$ for females [3].

In a previous study [4], we provided a map of the standardized mortality ratio (SMR) for pancreatic cancer in the 47 prefectures of Japan. This SMR map was based on census data and vital statistics from 1998 to 2002, and it showed high death rates for both sexes in the Hokkaido and the Tohoku region. The SMRs for pancreatic cancer in 
Japan in 2000 had the same characteristics [5]. In the study reported here, we extended the observation period and attempted to identify regional and temporal trends in pancreatic cancer death rates in Japan. We also investigated curvatures from period trends in different eras as well as birth cohort trends, using age-period-cohort models for the 47 prefectures of Japan. By means of these analyses, we attempted to clarify one of the aspects of pancreatic cancer and contribute to further research.

\section{Materials and methods}

\section{Mortality data}

The mortality data for pancreatic cancer (ICD-8: 157, ICD9: 157, ICD-10: C25) in all 47 Japanese prefectures were obtained from CD-ROMs of the National Vital Statistics, Statistics and Information Department of the Minister's Secretariat of the Ministry of Health, Labor and Welfare, which granted us the permission to use the data. Deaths at 40 years of age and over stratified by sex and 5-year age groups for the period 1968-2002 were abstracted in our study, with the exception of Okinawa, where the data are only for the period 1973-2002 because of insufficient data.

Before beginning the statistical analysis, we categorized the mortality data into seven periods-1968-1972, 19731977, 1978-1982, 1983-1987, 1988-1992, 1993-1997, and 1998-2002 - and averaged them by 5 years in each period. These averages were used to calculate the age-specific death rates for ten 5-year age groups (40-44 to $85+$ years) by period. The population in the census year that fell in the middle of each period (1970, 1975, 1980, 1985, 1990, and 2000) was used as the denominator. The age-specific death rates in Okinawa in the period 1968-1972 were not calculated for the reason stated above.

Statistical methods

To construct the disease maps, we calculated the direct ageadjusted mortality rates for persons aged 40 years and older using the Japanese 1985 model population as the standard. We graded the mortality rates into the five levels provided on the web page of the Ministry of Health, Labour and Welfare [6] and calculated these levels as follows. First, the average and the standard deviation of the mortality rate in each period were calculated. Second, level 1 and level 5 were defined as the rates below the average beyond the standard deviation and the rates above the average beyond the standard deviation, respectively. Finally, dividing the rest of the range, including the average, into tertiles, levels 2, 3, and 4 were defined as intertertile range, in order of size.

In terms of the estimates of parameters from the ageperiod-cohort models, it is known that these cannot be uniquely estimated because of the exact linear dependency among the three factors [7]. Many solutions to this dependency have been proposed [8, 9]. Here, we used the estimable functions for age-period-cohort analyses provided by Tarone and Chu [10] to investigate the curvatures from the period and cohort trends for analyzing the direction and magnitude of two time trends. The age-periodcohort model used in our study is

$E_{i j k}=\alpha_{i}+\pi_{j}+\gamma_{k}$,

where $E_{i j k}$ is the logarithm of the mortality rate $R_{i j}=\frac{d_{i j}}{N_{i j}}\left(d_{i j}\right.$ : number of cancer deaths, $N_{i j}$ : number of persons at risk), and $\alpha_{i}$ is the effect of age group $i ; \pi_{j}$ is the effect of time period $j$; and $\gamma_{k}$ is the effect of birth cohort $k$. A unique set of parameter estimates for the model was obtained by setting constraints on the parameters by the maximum likelihood method, and regression programs that make use of a particular model sometimes select these constraints arbitrarily. With respect to the arbitrariness, Clayton and Shifflers [11] showed that changes of parameters were uniquely estimated regardless of any constraints. Tarone and Chu [10] extended the estimable function of Clayton and Shifflers and developed a methodology for testing curvatures from trend between two successive groups of cohorts. The Tarone and Chu approach is named a contrast and is also applicable to trends in period [8].

In this study the populations were divided into ten 5-year age groups (40-44 to 85+ years), and time was divided into seven 5-year periods (1968-1972 to 1998-2002). As a result, we obtained 16 overlapping birth cohorts of 10 years each (1878-1887 to 1953-1962). Since there is the relationship among three indices: $k=j-i+10$.

We used the following estimable function [8, 10] to investigate curvatures from the period trend.

$C_{1}=\pi_{7}-\pi_{5}-\left(\pi_{4}-\pi_{2}\right)$,

where $C_{1}$ is the contrast for comparing the period trend in 1988-2002 with the period trend in 1973-1987. This function was designed to enable an approximate comparison between the group from 1990 onward and the group before 1990. To investigate curvatures from the cohort trend, we grouped the birth cohorts into an early cohort (1888-1897 to 1903-1912), a middle cohort (1913-1922 to 1928-1937), and a recent cohort (1938-1947 to 1953-1962).

The contrast $[8,10]$ between the middle cohort and early cohort can be estimated by $C_{2}=3 \gamma_{11}+\gamma_{10}-\gamma_{9}-3 \gamma_{8}-\left(3 \gamma_{6}+\gamma_{5}-\gamma_{4}-3 \gamma_{3}\right)$.

Similarly, the contrast between the recent cohort and middle cohort can be estimated by $C_{3}=3 \gamma_{16}+\gamma_{15}-\gamma_{14}-3 \gamma_{13}-\left(3 \gamma_{11}+\gamma_{10}-\gamma_{9}-3 \gamma_{8}\right)$.

The particular parameter estimates were maximum likelihood estimates and obtained by using the generalized 
linear model for univariate analyses and SPSS ver. 12.0J software for Windows (SPSS, Chicago, IL). The parameter constraint was default (the last period effect and the last two cohort effects are zero). Contrast coefficient matrices defined by the SPSS software package were customized according to the hypotheses $C_{1}=0, C_{2}=0$, and $C_{3}=0$ and were analyzed by the $F$ test $[12,13]$. Two-sided $P$ values less than 0.05 were considered to be statistically significant.

\section{Results}

Mortality map

According to the direct age-adjusted mortality rates for pancreatic cancer, all prefectures showed increasing trends in both sexes during the observation period (these rates are not shown). The overall age-adjusted mortality rates for pancreatic cancer for the first period (1968-1972) to the seventh period (1998-2002) increased by $70.3 \%$ among males and by $68.3 \%$ among females. Figure 1 shows the mortality map based on the levels described in the Materials and methods. This map illustrates the following findings: (1) Iwate, even in the Tohoku region, tended to have the average national mortality rates, (2) in the west of Japan, in Tottori, a relatively high number of males had level 5, and (3) level 5 occurred in the northern Kyushu area several times between the second period, 1973-1977, and the fifth period, 1988-1992, but did not occur in the sixth period, 1993-1997, or the seventh period, 1998-2002.

In the light of level 1 and level 5, Yamaguchi and Okinawa, for males, and Yamaguchi, Kagoshima, and Okinawa, for females, had level 1 for five or more
Fig. 1 Direct age-adjusted mortality rates for pancreatic cancer in the 47 prefectures during the period 1968-2002. White level 1, yellow level 2, orange level 3, red level 4, black level 5 (see online version for color coding). Level 1 and level 5 were defined as the rates below the average beyond the standard deviation and the rates above the average beyond the standard deviation, respectively. Levels 2, 3, and 4 were defined as the rate of intertertile range within the standard deviation, in order of size. The maps at the top are for males, and the maps at the bottom are for females

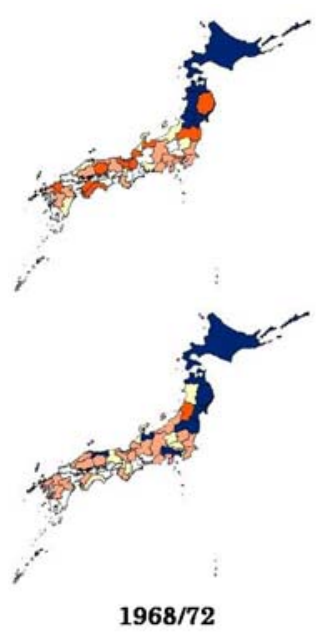

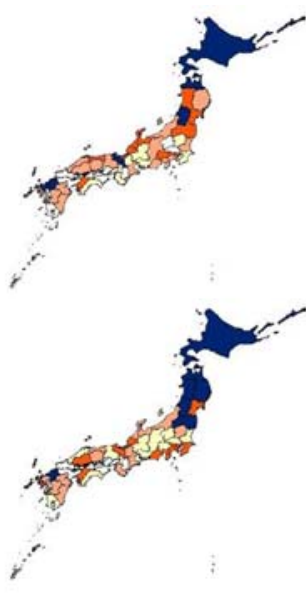

1973/77

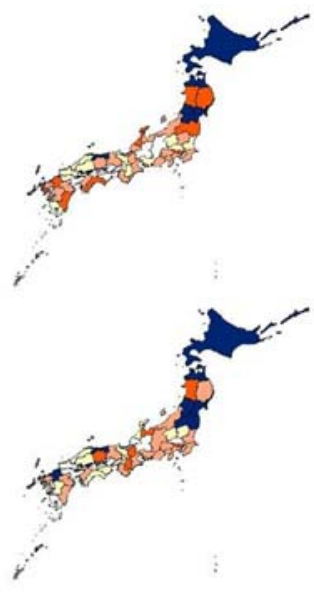

$1978 / 82$

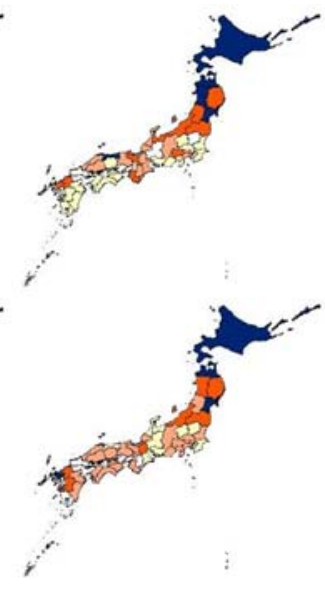

$1983 / 87$

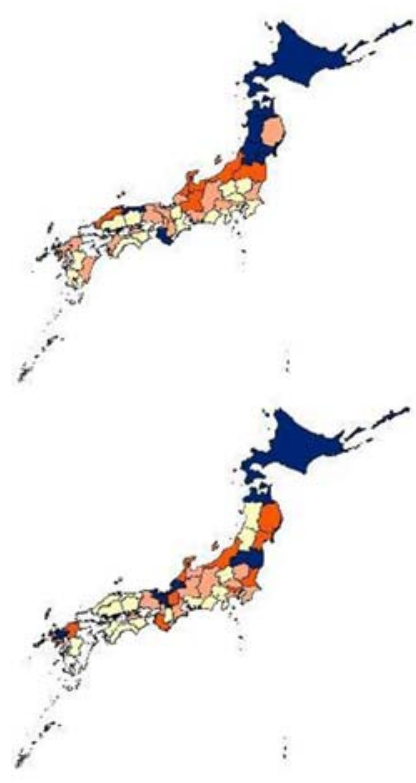

1988/92

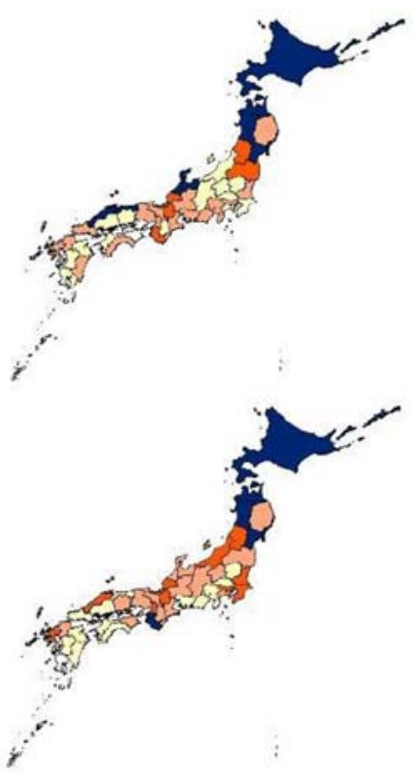

1993/97

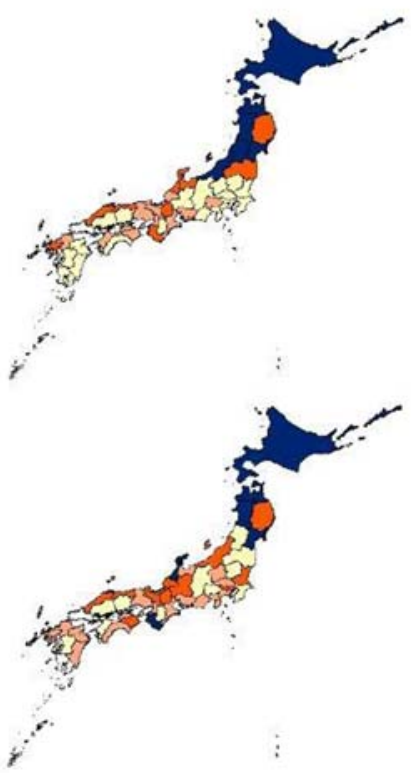

1998/2002 
successive times. Hokkaido, Aomori, Miyagi, Akita, and Yamagata, for males, and Hokkaido, Aomori, and Miyagi, for females, had level 5 for five or more successive times. Many level 5 regions were observed in Hokkaido and most of the Tohoku region during the observation period. Level 5 was identified in Hokkaido and Aomori in every period.

\section{Age-period-cohort models}

The model evaluation in each prefecture is shown in Table 1. Because we used the generalized linear model in this study, based on the assumption that the logarithm of the mortality rate is a linear function of regressor valuables, the goodness-of-fit of the age-period-cohort models was evaluated by residual sum of squares [13]. There were $40 d f$, except in Okinawa, where there were only $32 d f$ because of the lack of mortality data for the first period, 1968-1972. The effect of period and cohort factors was evaluated by the $F$ test. The period effect was statistically significant for males in Miyagi, Akita, Gumma, Saitama, Chiba, Toyama, Shiga, Osaka, and Wakayama, and for females in Hokkaido, Miyagi, Saitama, Fukui, and Yamanashi. The cohort effect was statistically significant for both sexes in all prefectures, with the exception in Shimane for males. Table 2 shows the results of the $F$ test for the estimable function $C_{1}$. Among the prefectures with bias toward level 1 or level 5, the estimated curvatures from the period trend $C_{1}$ decreased significantly in Miyagi and Kagoshima, for males, and in Hokkaido, for females. Hokkaido had the largest decrease in females: $C_{1}=-0.264(P=0.001)$. Among the prefectures with no bias toward level 1 and level 5, Iwate, Saitama, Chiba, Osaka, Nara, and Wakayama showed a significant decrease in $C_{1}$ for males. Wakayama had the largest decrease in males: $C_{1}=-0.590(P=0.003)$. The $C_{1}$ values showed that curvatures from the period trends between 1988-2002 and 1973-1987 were small in every prefecture. Table 3 shows the results of the $F$ test for the estimable function $C_{2}$. Every prefecture that had a level 1 or level 5 five or more times in succession had a significant decrease in $C_{2}$. Aomori had the largest decrease, $C_{2}=-2.779(P<0.001)$ for males, and Okinawa had the largest decrease, $C_{2}=-5.275$ $(P<0.001)$ for females. All of the prefectures with no bias toward level 1 and level 5, with the exception of Tottori, for males, and Gifu, Tottori, and Miyazaki, for females, showed a significant decrease in $C_{2}$. Toyama had the largest decrease in males, $C_{2}=-4.078(P<0.001)$, and Akita had the largest decrease in females, $C_{2}=-4.560(P<0.001)$. The $C_{2}$ values showed a decrease in cohort trends between 19131937 and 1888-1912 in most of the 47 prefectures. Table 4 shows the results of the $F$ test for the estimable function $C_{3}$. Among those prefectures with a bias toward level 1 and level 5 , the $C_{3}$ values showed a significant increase between 1938-1962 and 1913-1937 in Okinawa for both sexes, and a significant decrease in Hokkaido for both sexes. Yamaguchi had the largest decrease for males, $C_{3}=-2.826$ $(P=0.002)$, and Hokkaido had the largest decrease for females, $C_{3}=-1.057(P<0.032)$. Hokkaido and Okinawa showed a clear contrast between the age-adjusted mortality rate and the estimated curvatures from the cohort trends $C_{3}$. Among the prefectures with no bias toward level 1 or level 5, Kochi had the largest increase for males, $C_{3}=2.835$ $(P=0.002)$, and Shiga had the largest increase for females, $C_{3}=4.054(P<0.006)$. Tochigi had the largest decrease for males, $C_{3}=-4.342(P<0.001)$, and Ehime had the largest decrease for females, $C_{3}=-4.485(P<0.001)$. Based on the $C_{3}$ values between 1938-1962 and 1913-1937, curvatures from the cohort trends appeared to be different overall.

\section{Discussion}

The geographical trend in pancreatic cancer mortality in Japan that we detected in our study is consistent with that reported by Kato et al. [14]. Our previous SMR analysis of the 1998-2002 data showed the same geographical trend. The study reported here also showed the time trend of the mortality described in the Results.

Using an age-period-cohort analysis, we investigated the curvatures from the period and cohort trends for analyzing the direction and magnitude of two time trends in accordance with to Tarone and Chu's suggested use of estimable functions $C_{1}, C_{2}$, and $C_{3}$ These functions include information on whether the trends are concave upward or downward [12]. $C_{1}$ was used to investigate the curvatures from the period trends in the 1990s onwards. $C_{2}$ was used to investigate curvatures from the cohort trends between the early cohort (1888-1897 to 1903-1912) and the middle cohort (1913-1922 to 1928-1937), while $C_{3}$ was used to investigate curvatures from the cohort trends between the middle cohort and the recent cohort (1938-1947 to 1953-1962). These functions are the comparisons of period-based or cohortbased trends in different eras, but it is clear that they do not show direct associations with increases or decreases in pancreatic cancer mortality rates [8]. Furthermore, Tarone and Chu's approach was based on prior hypotheses which determined the groups of cohorts in female breast cancer mortality rates to be investigated $[10,15]$. In our study we did not conduct a survey of the grouping of periods and cohorts in each prefecture and, consequently, the validity of the grouping is a limitation of this study.

We did not observe any statistically significant changes in period trend between two groups in most of the prefectures. The classification code was changed three times during the observation periods, but there were no major changes in the classification of causes of pancreatic cancer 


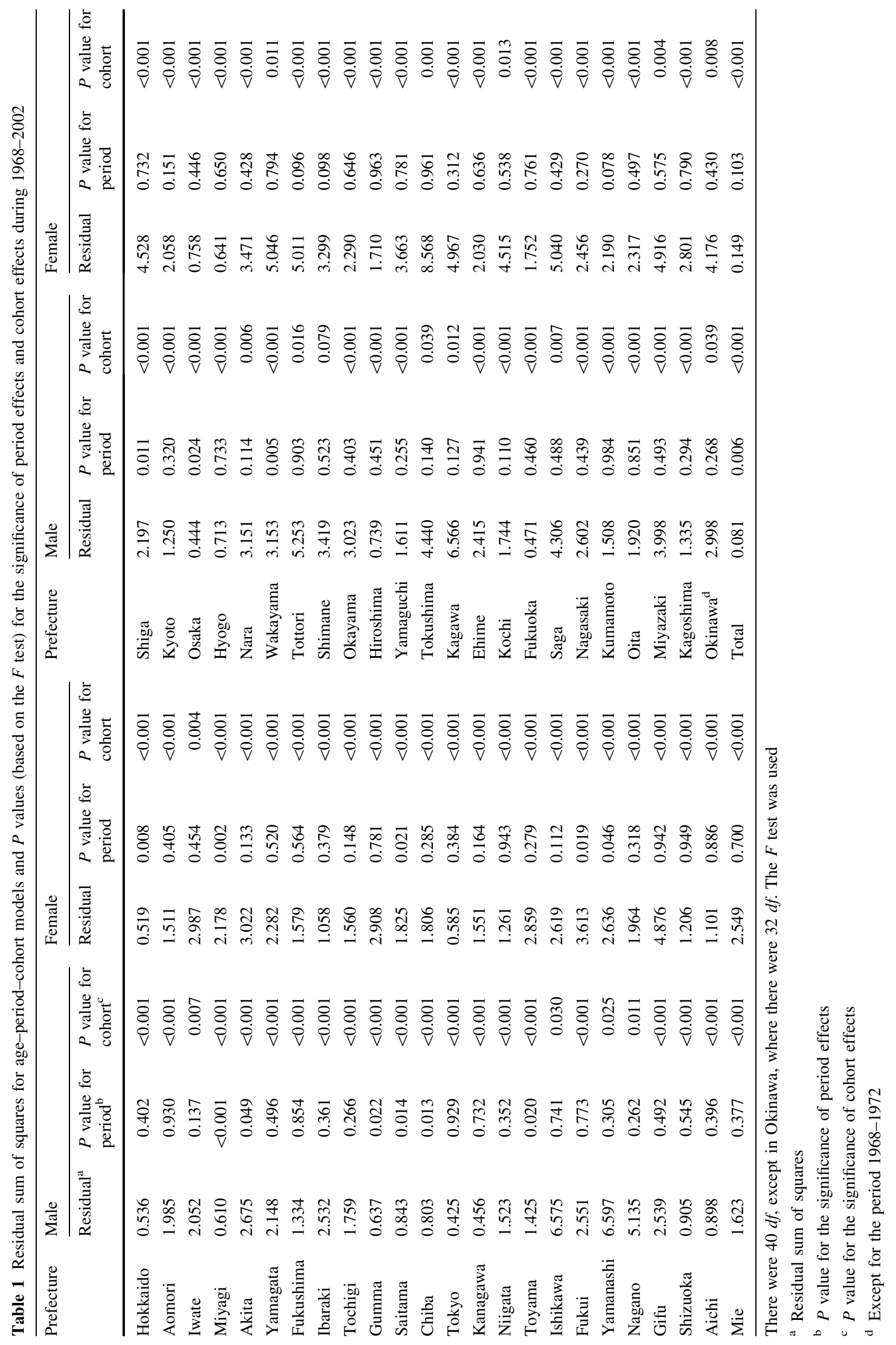




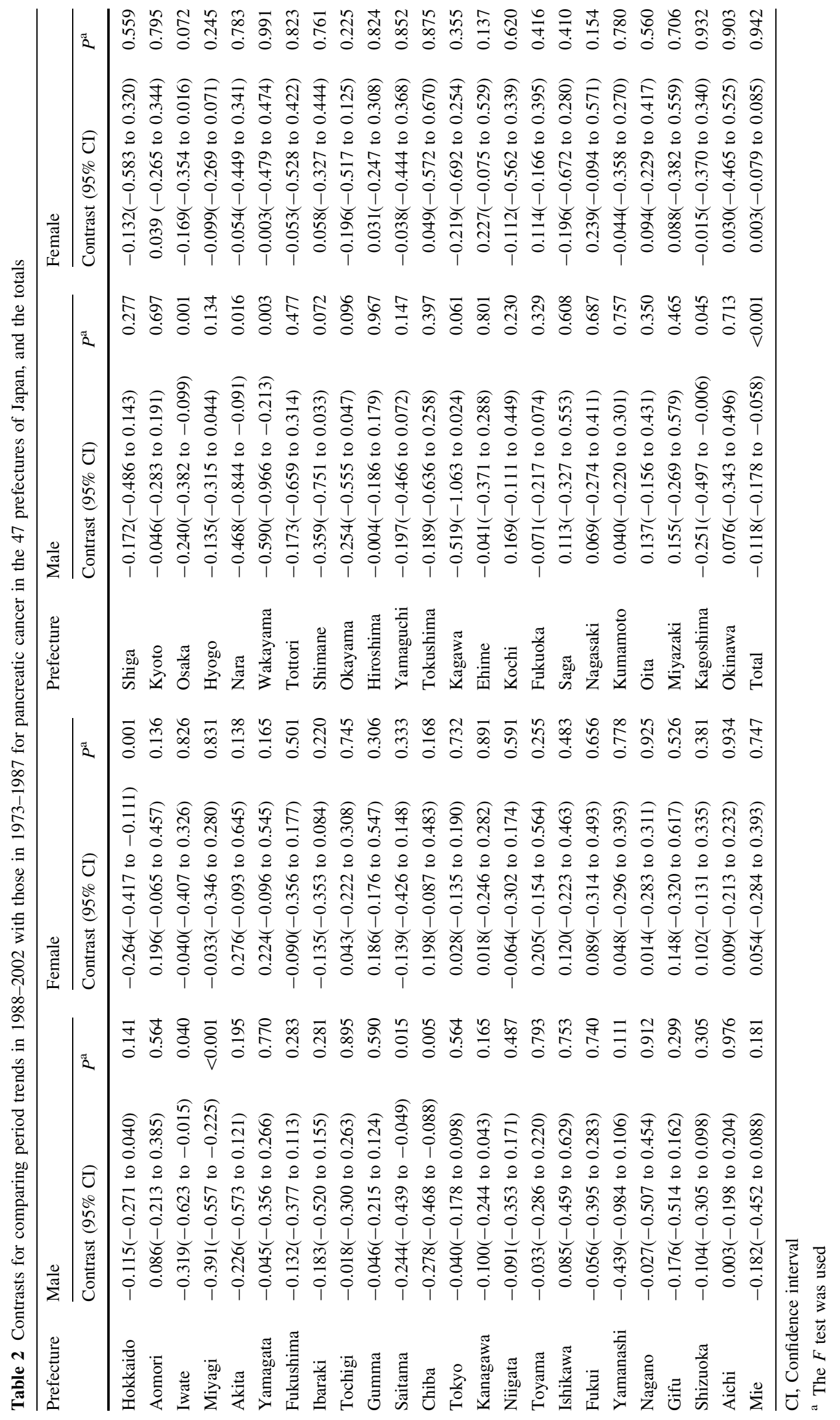




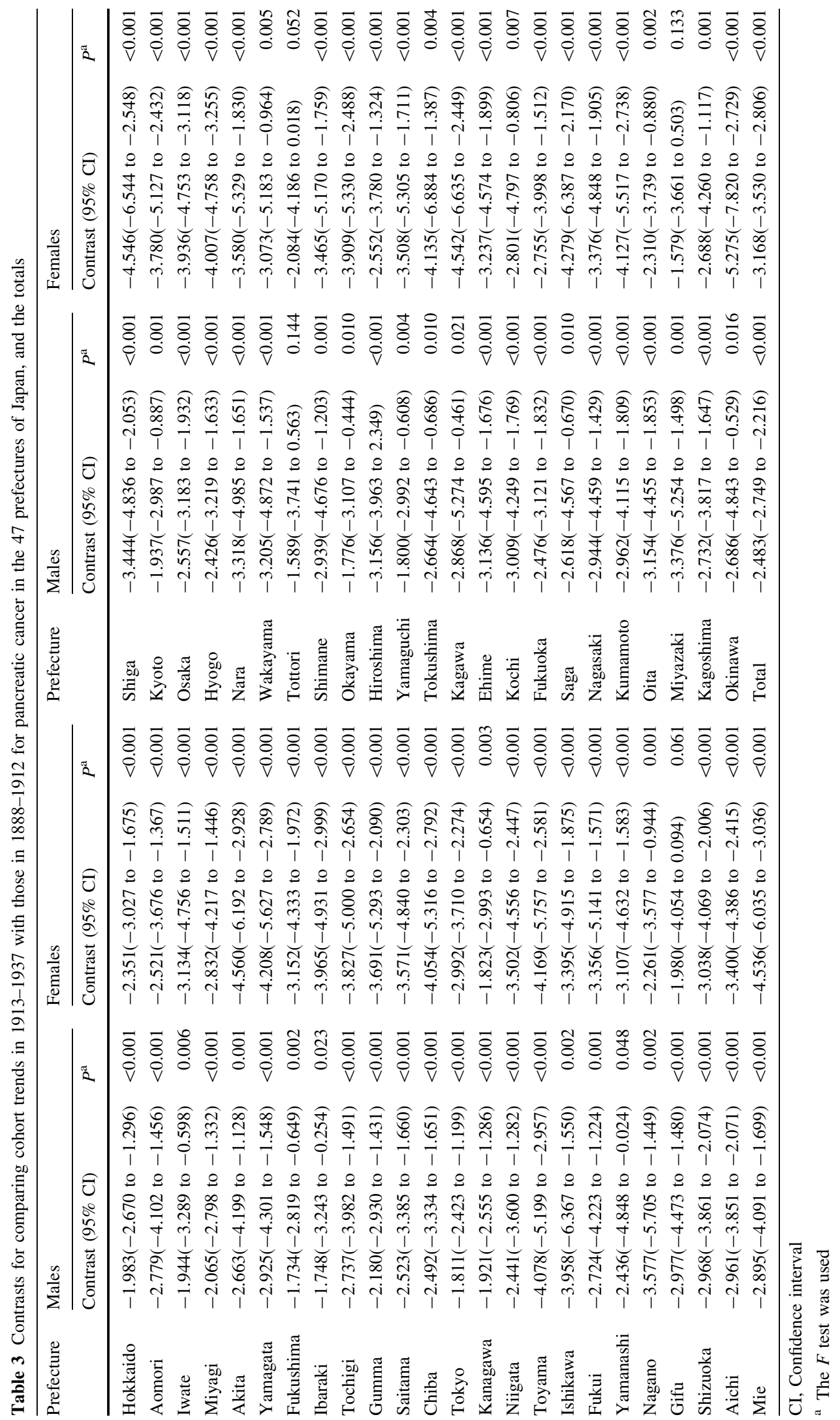




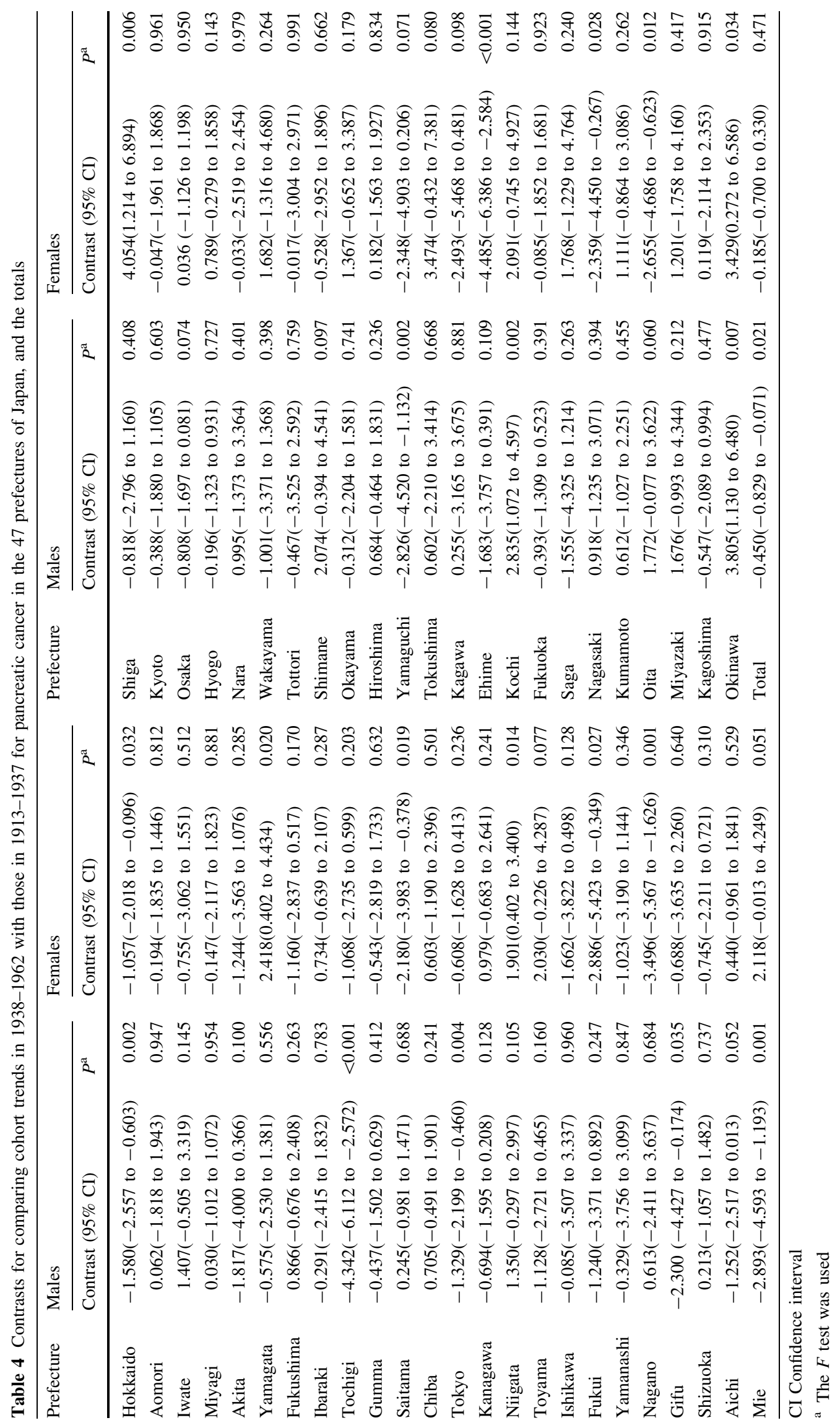


death. Changes in diagnostic and detection techniques or treatment are period factors that would affect all ages simultaneously [16]. These factors, however, were considered to have a minimal influence on the number of deaths in our analysis. On the other hand, almost all prefectures showed a statistically significant decline in cohort trend between the middle cohort and the early cohort, which would be more of an age effect than a cohort effect, partly because nearly $80 \%$ of the patients with pancreatic cancer were between 60 and 80 years of age [17]. In contrast, each prefecture showed a different change in cohort trend between the recent cohort and the middle cohort. There was a statistically significant increase in Okinawa and Kochi, for males, and in Yamagata, Niigata, Shiga, and Okinawa, for females. Okinawa had level 1 six successive times, and so this finding may mean that the generation gap in terms of mortality is becoming smaller between Okinawa and the other prefectures in recent years. However, it is not yet certain what sorts of cohort-related factor are involved. Long-term habits and long-term exposures are associated with cohort effects that result in different generations being exposed to different risks [9]. Several environmental factors affecting cohort effects have been presented [16], and one of the suspected risks among different generations is dietary habit. High cholesterol intake has been indicated as being related to the increment of pancreatic cancer risk according to population-based case-control study in Japan [18]. The westernization of the Japanese diet in the younger generations may partly account for the increase in pancreatic cancer over the past decades [19].

Tango et al. [20] considered the possibility of an ageperiod and an age-cohort interaction in age-period-cohort models and advocated period or cohort effects being constant within certain age groups. They adopted the conventional grouping: young age (20-34 years), middle age (35-54 years), and advanced age (55-79 years). In their study, the parameters of the models were estimated close between the middle age and the advanced age [20]; our present study incorporated the middle age into the advanced age. However the influence of an age interaction on period or cohort trends was not as clear as the two situation of the age groups.

In conclusion, this study carried out a combination of time trend analyses in mortality rates and the evaluation of changes in period or cohort trend based on estimable functions. The results reveal variations in mortality from pancreatic cancer from a different perspective in each prefecture. Further study is required to improve our statistical methods, and we need to continue to analyze pancreatic cancer mortality based on time effects.

Acknowledgments The Statistics and Information Department of the Minister's Secretariat of the Ministry of Health, Labour and Welfare provided us with a copy of the pancreatic cancer mortality data for the
47 prefectures. Prof. H. Suzuki and Mr. Y. Shobugawa of the Niigata University Human Health GIS Center helped design the mortality map of Japan, and we are grateful to them for their help and support.

\section{References}

1. Nomura S. The health of workers (39), pancreatic cancer and chemicals exposure to professions. (in Japanese). Rodo Kagaku. 2001;56:28-9.

2. Statistics and Information Department, Minister's Secretariat, Ministry of Health, Labour Minister's Secretariat, Ministry of Health, Labour and Welfare (in Japanese). Tokyo 2001.

3. Japan Cancer Association. The number of age specific deaths and rates in 2003 (in Japanese). Bull Jpn Cancer Assoc. 2004;488:46.

4. Seino T, Nakadaira H, Endoh K, Yamoto M. Descriptive epidemiologic studies on geographical clustering of pancreatic cancer mortality and incidence-an analysis in Japan and Niigata. Nippon Eiseigaku Zasshi. 2006;61:366-74.

5. Ohsima A, Isiguro T, Tazima K. White paper on cancer statistics-morbidity/mortality/prognosis-2004 (in Japanese). Tokyo: Sinoharasjupansinsja. 2004:82-3.

6. Ministry of Health, Labour and Welfare, Japan. Death aspects among the prefectures (in Japanese). Available from: http:// www.mhlw.go.jp/toukei/saikin/hw/jinkou/other/05sibou/01.html.

7. Holford TR. Understanding the effects of age, period, and cohort on incidence and cohort on incidence and mortality rates. Annu Rev Public Health. 1991;12:425-57.

8. Robertson C, Boyle P. Age-period-cohort analysis of chronic disease rates. I. Modeling approach. Stat Med. 1998;17:1305-23.

9. Robertson C, Gandini S, Boyle P. Age-period-cohort models: a comparative study of available methodologies. J Clin Epidemiol. 1999;52:569-83.

10. Tarone RE, Chu KC. Evaluation of birth cohort patterns in population disease rates. Am J Epidemiol. 1996;143:85-91.

11. Clayton D, Schifflers E. Models for temporal variation in cancer rates. II: Age-period-cohort models. Stat Med. 1987;6:469-81.

12. Holford TR. Age-period-cohort analysis. In: Mitchell HG, Benichou J, editors. Encyclopedia of epidemiologic methods. New York: Wiley; 2000. p. 17-35.

13. SPSS Inc. SPSS 12.0 Command Syntax Reference. Chicago, IL, USA: SPSS Inc.; 2003.

14. Kato I, Kuroish T, Tominaga S. Descriptive epidemiology of studies of cancers of the liver, biliary tract and pancreas in Japan. Jpn J Clin Oncol. 1990;20:232-7.

15. Tarone RE, Chu KC. Implication of birth cohort patterns in interpreting trends in breast cancer rates. J Natl Cancer Inst. 1992;84:1402-10.

16. Lin Y, Tamakoshi A, Wakai K, Kawamura T, Aoki R, Kojima M, et al. Descriptive epidemiology of pancreatic cancer in Japan. J Epidemiol. 1998;8:52-9.

17. Qiu D, Kurosawa M, Lin Y, Inada Y, Matsuba T, Kikuchi S, et al. Overview of the epidemiology of pancreatic cancer focusing on the JACC study. J Epidemiol. 2005;15[Suppl 2]:S157-67.

18. Lin Y, Tamakoshi A, Hayakawa T, Naruse S, Kitagawa M, Ohno Y. Nutritional factors and risk of pancreatic cancer: a populationbased case-control study based on direct interview in Japan. J Gastroenterol. 2005;40:297-301.

19. Lin Y, Kikuchi S, Tamakoshi A, Yagyu K, Obata Y, Inaba Y, et al. Dietary habits and pancreatic cancer risk in a cohort of middle-aged and elderly Japanese. Nutr Cancer. 2006;56(1):40-9.

20. Tango T, Kurashina S. Age, period and cohort analysis of trends in mortality from major disease in Japan, 1995 to 1978: peculiarity of the cohort born in the early Showa era. Stat Med. 1987;6:709-26. 